\title{
The Antibiotic Resistance Profile of Pseudomonas Aeruginosa in a Tertiary Medical Center from Malaysia
}

\author{
Mohd Ali Faiz ${ }^{1 *}$, Chuan Hun Ding ${ }^{2 *}$, Asrul Abdul Wahab², Mohd Nizam Tzar², Anita Sulong ${ }^{2}$, Kon Ken Wong², \\ Ping Foo Wong ${ }^{3}$
}

\begin{abstract}
Background: Pseudomonas aeruginosa is a notorious gram-negative bacterium that has become a global public health concern owing to the emergence of multi- and pandrug-resistant strains. This study sought to determine the antibiotic susceptibility profile of $P$. aeruginosa in a tertiary medical center from Malaysia.

Materials and Methods: Each isolate's identity was confirmed using the VITEK 2 GN kit, and subjected to antibiotic susceptibility testing using the VITEK 2 AST-N374 card (for testing against piperacillintazobactam, ceftazidime, cefepime, imipenem, meropenem, amikacin, gentamicin and ciprofloxacin) and Etest strips (for testing against doripenem and polymyxin B). Isolates which were not susceptible to $>$ I carbapenem were tested for carbapenemase production using the modified carbapenem inactivation method (mCIM).

Results: Out of 102 isolates studied, 64 (62.7\%) were fully susceptible to all the antibiotics tested. Twenty-six (25.5\%) were resistant to $>$ I antibiotic from $>2$ antibiotic classes, and 21 (20.6\%) were resistant to $>$ I antibiotic from $>3$ classes. Susceptibility was highest with polymyxin $B(100 \%)$ and lowest with piperacillin-tazobactam (64.7\%). Carbapenem susceptibility was between $78.4 \%$ to $81.4 \%$. Out of 22 isolates which were not susceptible to $>$ I carbapenem, 18 (8I.8\%) were not susceptible to all three carbapenems.

Conclusion: More than half of our P. aeruginosa isolates were fully susceptible to all the anti-pseudomonal antibiotics tested. Multidrug-resistant strains accounted for between $20 \%$ to $25 \%$ of all our P. aeruginosa isolates. Through $\mathrm{mCIM}$ testing, carbapenemase production did not appear to be the dominant resistance mechanism.

Keywords: Carbapenem, multidrug resistance, piperacillin-tazobactam, polymyxin B, Pseudomonas aeruginosa

DOI: https://doi.org/l0.3329/jom.v23il.57938 Copyright: (C) 2022 Faiz MA. This is an open access article published under the Creative Commons Attribution-NonCommercial-NoDerivatives 4.0 International License, which permits use, distribution and reproduction in any medium, provided the original work is properly cited, is not changed in any way and it is not used for commercial purposes.
\end{abstract}

Received: 12 August, 202I;

Accepted: 02 November, 2021

\section{Introduction}

Pseudomonas aeruginosa is an ancient bacterium first described more than a century ago by Migula in $1894 .{ }^{1}$ It is a ubiquitous non-fermenting and motile gram-negative bacterium easily recognized from its characteristic blue-green pigment. This bacterium is not usually considered part of normal human flora, but owing to its inherent capability to survive on minimal nutritional requirements and produce biofilms, it is able to withstand harsh environmental conditions which has facilitated its persistence in hospital settings. ${ }^{2,3}$ The gamut of diseases attributable to $P$. aerugionosa is extensive - it is implicated as a causative agent of serious infections such as bacteremia (often in association with neutropenia or vascular catheterization),

1. Medical Officer, Department of Medical Microbiology \& Immunology, Faculty of Medicine, Universiti Kebangsaan Malaysia, Kuala Lumpur, Malaysia

2. Clinical Microbiologist, Department of Medical Microbiology \& Immunology, Faculty of Medicine, Universiti Kebangsaan Malaysia, Kuala Lumpur, Malaysia

3. Family Medicine Physician, Cheras Baru Health Clinic, Health Department of Federal Territory of Kuala Lumpur and Putrajaya, Ministry of Health of Malaysia, Kampung Cheras Baru, Kuala Lumpur, Malaysia

*Corresponding author: Chuan Hun Ding, Associate Professor of Medical Microbiology, Department of Medical Microbiology \& Immunology, Faculty of Medicine, Universiti Kebangsaan Malaysia, 56000 Kuala Lumpur, Malaysia. Email: dingch@ppukm.ukm.edu.my 
pneumonia (particularly in mechanically ventilated patients), urinary tract infections (chiefly in patients with indwelling urinary catheters or stents), as well as skin and soft tissue infections (especially in the setting of skin burns or trauma). Moreover, $P$. aeruginosa infections have a propensity to become chronic and hard-to-eradicate. ${ }^{3}$

In 2008, a group of organisms was dubbed "the ESKAPE bugs" due to their legendary ability to escape the action of antimicrobial agents, with the "P" essentially referring to $P$. aeruginosa. ${ }^{4}$ P. aeruginosa is regarded as an "ESKAPE bug" due to the presence of various antibiotic resistance mechanisms in this versatile bacterium. The bacterium may be endowed with low outer-membrane permeability to antimicrobial agents, various drug efflux pumps, or antibioticinactivating enzymes. These mechanisms may even be present concurrently to confer multidrug resistance. ${ }^{5}$ Antimicrobial susceptibility testing is therefore essential in the management of $P$. aeruginosa infections. ${ }^{5}$ Local institutional data on the antimicrobial susceptibility profile (antibiogram) of this bacterium will assist clinicians in choosing the most efficacious antibiotic for empirical treatment which may not necessarily be the most broadspectrum or most financially costly drug. Therefore, the aim of this study was to construct a $P$. aeruginosa antibiogram to assist the formulation of institutional treatment guidelines that address infections likely to be caused by $P$. aeruginosa.

\section{Materials and Methods}

Study design

This cross-sectional study over a period of 18 months was conducted from January 2020 until June 2021. Non-duplicate $P$. aeruginosa isolates were collected from sterile specimens (e.g., blood, tissue, bone and body fluids) of patients who presented to Hospital Canselor Tuanku Muhriz (HCTM) in the capital city of Malaysia for various medical and surgical conditions.

Isolate identification

Any bacterium that produced a diffusible green, blue or bluegreen pigment on routine bacteriological media was subjected to an oxidase test. The oxidase test was performed using a filter paper disk moistened with a freshly prepared $1 \%$ solution of tetramethyl-p-phenylenediamine dihydrochloride. The ability of the tested organism (grown on either blood or Mueller Hinton agar) to cause the oxidase reagent to turn from colourless to dark purple within 10 seconds of being smeared was regarded as oxidase-positive.

To confirm the identity of $P$. aeruginosa, a commercial biochemical identification kit for gram-negative bacteria, VITEK 2 GN (bioMerieux, Inc., USA), was utilized. The biochemical identification was performed as per the manufacturer's protocol. A VITEK 2 GN identification percentage of at least $85 \%$ was taken as evidence of correct identification. Biochemical identification was sought because other pseudomonads (e.g., Pseudomonas fluorescens) may also produce pigments which are visually similar to that released by $P$. aeruginosa.

Antibiotic susceptibility testing

Once pure growths of young (18-24 hours old) P. aeruginosa colonies were obtained, the isolates were subjected to antibiotic susceptibility testing using the VITEK 2 AST-N374 card for gram-negative bacteria (bioMerieux, Inc., USA), as per the manufacturer's instructions. The card provided the antibiotic minimal inhibitory concentrations (MICs) for, among others, piperacillin-tazobactam, ceftazidime, cefepime, imipenem, meropenem, amikacin, gentamicin and ciprofloxacin. All MIC values were interpreted according to the 2019 edition of the Clinical \& Laboratory Standards Institute (CLSI) document M1006).

Whenever the VITEK 2 AST-N374 card reported a carbapenem-resistant MIC, the isolate's MIC to doripenem and polymyxin B (which were not included in the card's panel) were determined using relevant Etest strips (bioMerieux SA, France), in accordance to the manufacturer's instructions. Before reading the MIC values, the Mueller Hinton (MH) agar plates containing the Etest strips were visually inspected to ensure no contamination had occurred during incubation and that a confluent bacterial lawn had been achieved. The MIC for each antibiotic was read by recording the point at which the inhibition ellipse intersected the side of the Etest strip.

\section{Detection of carbapenemase production}

A modified carbapenem inactivation method (mCIM) was perfomed as described by the CLSI whenever a $P$. aeruginosa isolate had either an intermediate or resistant MIC (i.e., nonsusceptible) to even a single carbapenem agent. Essentially, for each $P$. aeruginosa isolate, a $10 \mathrm{ml}$ inoculum obtained from a pure overnight colony was suspended in $2 \mathrm{ml}$ of trypticase soy broth (TSB). A $10 \mathrm{~g}$ meropenem disk was added to the TSB suspension, followed by an incubation at $35^{\circ} \mathrm{C}$ in ambient air for 4 hours. Just before the completion of the TSB-meropenem disk incubation, a $0.5 \mathrm{McF}$ arland suspension of a carbapenem-susceptible indicator organism (Escherichia coli ATCC $^{\mathrm{O}}$ 25922) was prepared in saline and seeded onto an $\mathrm{MH}$ plate within 15 minutes. After allowing the inoculated $\mathrm{MH}$ agar plate to dry for 5-10 minutes, the meropenem disk from the TSB suspension was removed and placed on the MH agar plate inoculated with E. coli. The 
meropenem disk-containing $\mathrm{MH}$ agar plate was then incubated at $35^{\circ} \mathrm{C}$ in ambient air for $18-24$ hours. The production of carbapenamase by a $P$. aeruginosa isolate was inferred either by a meropenem disk inhibition zone of $£ 15 \mathrm{~mm}$ in diameter or the presence of colonies within the inhibition zone, while the absence of carbapenemase was indicated by meropenem inhibition zones of ${ }^{3} 19 \mathrm{~mm}$. Isolates which formed inhibition zones of between 16 and $18 \mathrm{~mm}$ were interpreted as indeterminate for carbapenemase production.

\section{Results}

Demographic data of study subjects

Table 1 shows the demographic data of study subjects. A total of 102 non-repetitive $P$. aeruginosa isolates from 102 different patients were included in the study. There were slightly more male patients $(52.9 \%)$ compared to female patients $(47.1 \%)$. The proportion of fully susceptible $P$. aeruginosa isolates was higher in the female gender $(69 \%$ vs. $57 \%)$. Most of the patients (30.4\%) were aged between 60-69 years, with the median age being 63 years. For most age groups, the proportion of fully susceptible $P$. aeruginosa isolates predominated. However, for the 50-59 years age group, half of the $P$. aeruginosa isolates were resistant to $>1$ antibiotic. Regarding ethnicity, most patients were Malays $(60.8 \%)$, followed by Chinese $(34.3 \%)$ and Indians $(2.9 \%)$. Irrespective the ethnicity, the percentage of fully susceptible $P$. aeruginosa isolates predominated.

Table I : Demographic data of study subjects

\begin{tabular}{|c|c|c|c|c|c|}
\hline Characteristic & $\begin{array}{c}\text { Total } \\
(\mathrm{n}=102)\end{array}$ & $\begin{array}{l}\text { Susceptible to } \\
\text { all antibiotic } \\
\text { agents } \\
(n=64)\end{array}$ & $\begin{array}{c}\text { Resistant to } \\
\text { at least one } \\
\text { antibiotic agent } \\
\quad(n=31)\end{array}$ & $\begin{array}{c}\text { Resistant to } \\
\text { at least one } \\
\text { agent from } \\
>2 \text { antibiotic classes } \\
(n=26)\end{array}$ & $\begin{array}{c}\text { Resistant to } \\
\text { at least one } \\
\text { agent from }>3 \\
\text { antibiotic classes } \\
(\mathrm{n}=21)\end{array}$ \\
\hline \multicolumn{6}{|l|}{ Gender } \\
\hline Male & $54(52.9 \%)$ & $31(48.4 \%)$ & $18(58.1 \%)$ & $16(61.5 \%)$ & $13(61.9 \%)$ \\
\hline Female & $48(47.1 \%)$ & $33(51.6 \%)$ & $13(41.9 \%)$ & $10(38.5 \%)$ & $8(38.1 \%)$ \\
\hline \multicolumn{6}{|l|}{ Age group (yr) } \\
\hline$<9$ & $6(5.9 \%)$ & $6(9.4 \%)$ & $0(0.0 \%)$ & $0(0.0 \%)$ & $0(0.0 \%)$ \\
\hline $10-19$ & $0(0.0 \%)$ & $0(0.0 \%)$ & $0(0.0 \%)$ & $0(0.0 \%)$ & $0(0.0 \%)$ \\
\hline $20-29$ & $5(4.9 \%)$ & $4(6.2 \%)$ & $1(3.2 \%)$ & $1(3.9 \%)$ & $1(4.8 \%)$ \\
\hline $30-39$ & $4(3.9 \%)$ & $2(3.1 \%)$ & $1(3.2 \%)$ & $1(3.9 \%)$ & $1(4.8 \%)$ \\
\hline $40-49$ & $12(11.8 \%)$ & $9(14.1 \%)$ & $3(9.7 \%)$ & $3(11.5 \%)$ & $3(14.3 \%)$ \\
\hline $50-59$ & $12(11.8 \%)$ & $5(7.8 \%)$ & $5(16.1 \%)$ & $4(15.4 \%)$ & $4(19.0 \%)$ \\
\hline $60-69$ & $31(30.4 \%)$ & $15(23.4 \%)$ & $14(45.2 \%)$ & $11(42.3 \%)$ & $8(38.1 \%)$ \\
\hline $70-79$ & $25(24.5 \%)$ & $17(26.6 \%)$ & $7(22.6 \%)$ & $6(23.1 \%)$ & $4(19.0 \%)$ \\
\hline$>80$ & $7(6.9 \%)$ & $6(9.4 \%)$ & $0(0.0 \%)$ & $0(0.0 \%)$ & $0(0.0 \%)$ \\
\hline \multicolumn{6}{|l|}{ Ethnicity } \\
\hline Malay & $62(60.8 \%)$ & $37(57.8 \%)$ & $20(64.5 \%)$ & $18(69.2 \%)$ & $15(71.4 \%)$ \\
\hline Chinese & $35(34.3 \%)$ & $22(34.4 \%)$ & $11(35.5 \%$ & $8(30.8 \%)$ & $6(28.6 \%)$ \\
\hline Indian & $3(2.9 \%)$ & $3(4.7 \%)$ & $0(0.0 \%)$ & $0(0.0 \%)$ & $0(0.0 \%)$ \\
\hline Foreigner & $2(2.0 \%)$ & $2(3.1 \%)$ & $0(0.0 \%)$ & $0(0.0 \%)$ & $0(0.0 \%)$ \\
\hline
\end{tabular}




\section{Antibiotic susceptibility profile of isolates}

Out of 102 isolates, $64(62.7 \%)$ were fully susceptible to all the 10 antibiotics from a total of six antibiotic classes, viz., anti-pseudomonal penicillins, anti-pseudomonal cephalosporins, carbapenems, fluoroquinolones, aminoglycosides and polymyxins. Thirty-eight (37.3\%) isolates were resistant to at least one antibiotic agent. Twenty-six (25.5\%) isolates were resistant to at least one agent from $>2$ antibiotic classes, and $21(20.6 \%)$ were resistant to at least one agent from $>3$ classes (Table 1). As shown in Table 2, non-susceptibility was highest with the antipseudomonal penicillin class (typified by piperacillintazobactam), followed by the cephalosporin (represented by ceftazidime and cefepime), the carbapenem (represented by meropenem, imipenem and doripenem), the fluoroquinolone (typified by ciprofloxacin), and the aminoglycoside (represented by gentamicin and amikacin) classes. None of the isolates were resistant to polymyxin B. For each antibiotic tested, $50 \%$ of the isolates could be inhibited by an MIC which was low enough to be interpreted as susceptible, although (with the exception of polymyxin B) only an MIC which was high enough to be interpreted as resistant could inhibit $90 \%$ of our isolates.

As presented in Table 3, out of 22 P. aeruginosa isolates which were not susceptible (i.e., testing either intermediate or resistant) to at least one carbapenem, 18 (81.8\%) were non-susceptible to all three carbapenems tested and four were still susceptible to at least one carbapenem class member. The mCIM test was only found to be positive in one-third of the pan-carbapenem-non-susceptible isolates and negative in all the carbapenem-non-susceptible isolates which were still susceptible to at least one carbapenem agent. There was no statistically significant association between the extent of carbapenem non-susceptibility and mCIM positivity.

Table 2 : Antibiotic susceptibility profile of Pseudomonas aeruginosa

\begin{tabular}{|c|c|c|c|c|c|}
\hline Antibiotic & $\begin{array}{c}\text { Susceptible, } \\
\text { no. }(\%)\end{array}$ & $\begin{array}{c}\text { Intermediate, } \\
\text { no. }(\%)\end{array}$ & $\begin{array}{c}\text { Resistant, } \\
\text { no. }(\%)\end{array}$ & $\begin{array}{l}\mathrm{MIC}_{50} \\
(\mu \mathrm{g} / \mathrm{ml})\end{array}$ & $\begin{array}{l}\mathrm{MIC}_{90} \\
(\mu \mathrm{g} / \mathrm{ml})\end{array}$ \\
\hline Piperacillin-tazobactam & $66(64.7)$ & $8(7.8)$ & $28(27.5)$ & 8 & e"128* \\
\hline Ceftazidime & $74(72.5)$ & $2(2.0)$ & $26(25.5)$ & 2 & e"64* \\
\hline Cefepime & $76(74.5)$ & $7(6.9)$ & $18(17.6)$ & 2 & e"32* \\
\hline Meropenem & $80(78.4)$ & $5(4.9)$ & $17(16.7)$ & 0.5 & e"16* \\
\hline Imipenem & $83(81.4)$ & $0(0.0)$ & $19(18.6)$ & 2 & e"16* \\
\hline Doripenem & $83(81.4)$ & $4(3.9)$ & $15(14.7)$ & 0.38 & e"32* \\
\hline Ciprofloxacin & $87(85.3)$ & $1(1.0)$ & $14(13.7)$ & 0.25 & e"4* \\
\hline Gentamicin & $88(86.3)$ & $0(0.0)$ & $14(13.7)$ & d"1 & e"16* \\
\hline Amikacin & $90(88.2)$ & $1(1.0)$ & $11(10.8)$ & 4 & e"64* \\
\hline Polymyxin B & $102(100.0)$ & $0(0.0)$ & $0(0.0)$ & 1.5 & 2 \\
\hline
\end{tabular}

MIC: minimal inhibitory concentration, $\mathrm{MIC}_{50}$ and $\mathrm{MIC}_{90}$ : antibiotic $\mathrm{MIC}$ capable of inhibiting $50 \%$ and $90 \%$ of isolates, respectively, *indicates resistant MIC

Table 3 : mCIM results for carbapenem-non-susceptible Pseudomonas aeruginosa

\begin{tabular}{lccc}
\hline Extent of carbapenem non-susceptibility & & mCIM result & \\
& Positive & Negative & p-value* \\
\hline All three carbapenems & $6(27.3 \%)$ & $12(54.5 \%)$ & 0.541 \\
Less than three carbapenems & $0(0.0 \%)$ & $4(18.2 \%)$ & \\
\hline
\end{tabular}

mCIM: modified carbapenem inactivation method, *derived from Fisher's exact test 


\section{Discussion}

Over the years, multidrug-resistant (MDR) P. aeruginosa has been increasingly isolated from hospitalized patients and is of particular concern in critically ill and immunocompromised patients. The global prevalence of MDR $P$. aeruginosa is now expected to be between $15 \%$ and $30 \%$ in multiple geographical areas. ${ }^{7}$ Drug-resistant $P$. aeruginosa infections give rise to higher mortality, higher morbidity, additional resource utilization and increased financial costs. A meta-analysis found that patients infected with any resistant $P$. aeruginos $a$ have an all-cause mortality of $34 \%$, compared to $22 \%$ for those infected with susceptible P. aeruginosa. ${ }^{8}$ The same meta-analysis also reported that there is at least a 2-fold increased risk of mortality with MDR P. aeruginosa. ${ }^{8}$ Of noteworthy concern is resistance to carbapenems, which can catapult the mortality rate to as high as $71 \% .{ }^{9}$ MDR P. aeruginos $a$ are also adept at spreading or transferring resistance determinants in vivo, particularly those encoding carbapenemases or extended-spectrum âlactamases. ${ }^{10}$ Thus, it is evident that knowing the prevalence of drug-resistant $P$. aeruginosa in any healthcare setting is no longer merely of academic interest.

Unfortunately, comparing prevalence or incidence data with other healthcare facilities is not so straightforward for the simple reason that the definition of "multidrug resistance" is inconsistent. Depending on the study or centre, "multidrug resistance" can either refer to resistance to at least one agent from $>2$ antibiotic classes, ${ }^{11,12}$ or resistance to at least one agent from $>3$ antibiotic classes. ${ }^{13-15}$ A study conducted in Hospital Kuala Lumpur (which is a healthcare facility located H"12 km away from HCTM) found that $19.6 \%$ of $P$. aeruginosa isolates were resistant to $>2$ antibiotic classes in 2007. ${ }^{11}$ One and a half decades later, by employing the same MDR definition as Pathmanathan et al., our rate of MDR P. aeruginosa appears to be higher at $25.5 \%$. However, by defining MDR $P$. aeruginos $a$ as resistance to $>3$ antibiotic classes, our centre's MDR P. aeruginosa frequency of $20.6 \%$ is still considerably lower compared to other major Asian nations, such as China (29.0\%) and India $(31.7 \%) .{ }^{13,14} \mathrm{We}$ are also fortunate to have no pandrug-resistant (defined as resistance to all anti-pseudomonal antibiotics, including polymyxins) $P$. aeruginosa isolates in our centre to date, although globally pandrug-resistant $P$. aeruginosa strains have already been reported in the literature for more than a decade. ${ }^{16}$

Our $P$. aeruginosa isolates were least susceptible to piperacillin-tazobactam, with a susceptibility rate of $64.7 \%$. This observation is in stark contrast to the Hospital Kuala Lumpur study which reported that piperacillin-tazobactam was the most active anti-pseudomonal antibiotic, with a susceptibility rate of $91.8 \% .{ }^{11}$ This difference in susceptibility could be due to variations in antibiotic prescribing practices between hospitals, which in turn result in different drug selection pressures being applied on the organism. For instance, if piperacillin-tazobactam is favored over cefepime for the empirical treatment of high-risk adult patients with neutropenic sepsis, one could expect a higher resistance rate to be recorded for the former antibiotic. With a susceptibility of $100 \%$, polymyxin B appeared to the best antibiotic against $P$. aeruginosa in our study, although we are cognizant that the in vitro susceptibility testing of polymyxin B is fraught with difficulties. ${ }^{17}$ The CLSI recommends only broth microdilution as the reference method to perform polymyxin B susceptibility testing, although we utilized an alternative method (i.e., Etest), akin to what Falagas et al. performed in their study. ${ }^{16}$ Notwithstanding the CLSI's recommendation, the Etest (which is fundamentally a gradient diffusion method) has been shown to correlate well with the reference broth microdilution method. ${ }^{18}$

Susceptibility testing issues aside, polymyxin B has been found to be mediocre to other anti-pseudomonal antibiotics, as signified by the higher rate of in-hospital mortality associated with its usage. ${ }^{19}$ Moreover, being the "antibiotic of last resort" in our antibiotic arsenal against $P$. aeruginosa (and other gram-negative pathogens), polymyxin B should be prescribed judiciously and sparingly. Some authorities advocate combination empirical therapy (sans the polymyxins) for severe $P$. aeruginosa infections, especially among critically ill patients. The rational for combination therapy is to enhance the prospect of selecting an effective antibiotic for empirical therapy, rather than to hamper resistance from developing during definitive therapy, or to obtain benefit from antibiotic synergism. ${ }^{9}$ Looking at our own data, agents from the aminoglycoside and fluoroquinolone classes (which have documented susceptibilities in excess of $85 \%$ ) are excellent pairing partners to the beta-lactam agents (including the carbapenems). ${ }^{20}$

The mechanisms conferring resistance to carbepenems can be expediently divided into carbapenemase-producing $(\mathrm{CP})$ and non-CP mechanisms. The former is particularly pertinent for infection control and epidemiologic reasons, because the carbapenemase genes are borne on mobile genetic elements (e.g., plasmids and transposons) that can be transmitted horizontally to other gram-negative organisms. ${ }^{21,22}$ Additionally, it is believed that $\mathrm{CP}$ bacteria are more virulent than their carbapenem-resistant but non$\mathrm{CP}$ counterparts. ${ }^{23}$ The mCIM and its predecessor (known 
simply as CIM) were initially developed to detect carbapenemase production in carbapenem-resistant Enterobacteriaceae. ${ }^{21}$ However, the mCIM has also been found to possess excellent sensitivity (98\%) and specificity (95\%) in identifying carbapenemase production in $P$. aeruginosa, and is now recommended by the CLSI for the same purpose. ${ }^{6,22}$ Consistent with the published literature that the most frequent mechanism of carbapenem resistance in $P$. aeruginosa is related to oprD porin mutations, it is not surprising that most of our own isolates with pan-carbapenem non-susceptibility were actually mCIM-negative. ${ }^{22}$ The fact that we found no statistically significant association between mCIM positivity and the extent of carbapenem resistance in our study essentially means that most of our P. aeruginosa with pan-carbapenem-resistant phenotypes are not $\mathrm{CP}$ bacteria, and thus do not pose infection control nightmares.

\section{Conclusions}

More than half of our center's $P$. aeruginosa isolates were still fully susceptible to all commonly used anti-pseudomonal antibiotics. MDR strains only accounted for, depending on the definition used, either $20 \%$ to $25 \%$ of all $P$. aeruginosa isolates. Antibiotic resistance was most evident with piperacillin-tazobactam and least pronounced with polymyxin $\mathrm{B}$, with the latter documenting zero resistance. Although most of our isolates were fully carbapenem-susceptible, slightly less than one-fifth were pan-carbapenem-nonsusceptible. Fortunately, mCIM testing showed that amongst our pan-carbapenem-non-susceptible $P$. aeruginosa isolates, carbapenemase production was not the dominant resistance mechanism.

\section{Acknowledgement}

We would like to thank the Faculty of Medicine, Universiti Kebangsaan Malaysia, for providing a research grant (code: FF-2019-531), without which this study would not have been possible. We are also grateful to the Dean, Faculty of Medicine, Universiti Kebangsaan Malaysia, for his support and permission to publish the findings from this study.

\section{Conflicts of interest: None}

\section{References}

1. Palleroni NJ. The Pseudomonas story. Environ Microbiol 2010; 12: 1377-83. https://doi.org/10.1111/j.14622920.2009.02041.x

2. Lister PD, Wolter DJ, Hanson ND. Antibacterial-resistant Pseudomonas aeruginosa: clinical impact and complex regulation of chromosomally encoded resistance mechanisms. Clin Microbiol Rev 2009; 22: 582-610. https://doi.org/ 10.1128/cmr.00040-09
3. Behzadi P, Baráth Z, Gajdács M. It's not easy being green: a narrative review on the microbiology, virulence and therapeutic prospects of multidrug-resistant Pseudomonas aeruginosa. Antibiotics 2021; 10: 42. https://doi.org/10.3390/ antibiotics 10010042

4. Rice LB. Federal funding for the study of antimicrobial resistance in nosocomial pathogens: no ESKAPE. J Infect Dis 2008; 197: 1079-81. https://doi.org/10.1086/533452

5. Mesaros N, Nordmann P, Plésiat P, Roussel-Delvallez M, Van Eldere J, Glupczynski Y, et al. Pseudomonas aeruginosa: resistance and therapeutic options at the turn of the new millennium. Clin Microbiol Infect 2007; 13: 560-78. https:/ /doi.org/10.1111/j.1469-0691.2007.01681.x

6. CLSI. Performance standards for antimicrobial susceptibility testing. 29th edition. CLSI supplement M100. Wayne, PA: Clinical and Laboratory Standards Institute, 2019.

7. Horcajada JP, Montero M, Oliver A, Sorlí L, Luque S, GómezZorrilla S, et al. Epidemiology and treatment of multidrugresistant and extensively drug-resistant Pseudomonas aeruginosa infections. Clin Microbiol Rev 2019; 32: e0003119. https://doi.org/10.1128/cmr.00031-19

8. Nathwani D, Raman G, Sulham K, Gavaghan M, Menon V. Clinical and economic consequences of hospital-acquired resistant and multidrug-resistant Pseudomonas aeruginosa infections: a systematic review and meta-analysis. Antimicrob Resist Infect Control 2014; 3: 32. https://doi.org/10.1186/ 2047-2994-3-32

9. Bassetti M, Vena A, Croxatto A, Righi E, Guery B. How to manage Pseudomonas aeruginosa infections. Drugs Context 2018; 7: 212527. https://doi.org/10.7573/dic.212527

10. Oliver A, Mulet X, López-Causapé C, Juan C. The increasing threat of Pseudomonas aeruginosa high-risk clones. Drug Resist Updat 2015; 21-22: 41-59. https://doi.org/10.1016/ j.drup.2015.08.002

11. Pathmanathan SG, Samat NA, Mohamed R. Antimicrobial susceptibility of clinical isolates of Pseudomonas aeruginosa from a Malaysian Hospital. Malays J Med Sci 2009; 16: 2732.

12. Mohd Nasir MD, Nurnajwa MH, Lay J, Teoh JC, Syafinaz AN, Niazlin MT. Risk factors for multidrug-resistant Pseudomonas aeruginosa among hospitalized patients at a Malaysian hospital. Sains Malaysiana 2015; 44: 257-260.

13. Prakash V, Mishra PP, Premi HK, Walia A, Dhawan S, Kumar A. Increasing incidence of multidrug resistant Pseudomonas aeruginosa in inpatients of a tertiary care hospital. Int J Res Med Sci 2014; 2: 1302-6. https://doi.org/10.5455/23206012.ijrms20141111

14. Peng Y, Shi J, Bu T, Li Y, Ye X, Chen X, et al. Alarming and increasing prevalence of multidrug-resistant Pseudomonas aeruginosa among healthcare-associated infections in China: 
A meta-analysis of cross-sectional studies. J Glob Antimicrob Resist 2015; 3: 155-160. https://doi.org/10.1016/ j.jgar.2015.04.001.

15. Sid Ahmed MA, Hassan AAI, Abu Jarir S, Abdel Hadi H, Bansal D, Abdul Wahab A, et al. Emergence of multidrugand pandrug- resistant Pseudomonas aeruginosa from five hospitals in Qatar. Infect Prev Pract 2019; 1: 100027. https:/ /doi.org/10.1016/j.infpip.2019.100027.

16. Falagas ME, Bliziotis IA, Kasiakou SK, Samonis G, Athanassopoulou P, Michalopoulos A. Outcome of infections due to pandrug-resistant (PDR) gram-negative bacteria. BMC Infect Dis 2005; 5: 24. https://doi.org/10.1186/ 1471-2334-5-24.

17. Ezadi F, Ardebili A, Mirnejad R. Antimicrobial susceptibility testing for polymyxins: challenges, issues, and recommendations. J Clin Microbiol 2019; 57: e01390-18. https://doi.org/10.1128/jcm.01390-18.

18. Behera B, Mathur P, Das A, Kapil A, Gupta B, Bhoi S, et al. Evaluation of susceptibility testing methods for polymyxin. Int J Infect Dis 2010; 14: e596-601. https://doi.org/10.1016/ j.ijid.2009.09.001.

19. Kvitko CH, Rigatto MH, Moro AL, Zavascki AP. Polymyxin $B$ versus other antimicrobials for the treatment of Pseudomonas aeruginosa bacteraemia. J Antimicrob
Chemother 2011; 66: 175-9. https://doi.org/10.1093/jac/ dkq390.

20. Tschudin-Sutter S, Fosse N, Frei R, Widmer AF. Combination therapy for treatment of Pseudomonas aeruginosa bloodstream infections. PLoS One 2018; 13: e0203295. https://doi.org/10.1371/journal.pone.0203295.

21. Pierce VM, Simner PJ, Lonsway DR, Roe-Carpenter DE, Johnson JK, Brasso WB, et al. Modified carbapenem inactivation method for phenotypic detection of carbapenemase production among Enterobacteriaceae. J Clin Microbiol 2017; 55: 2321-33. https://doi.org/10.1128/ jcm.00193-17.

22. Simner PJ, Johnson JK, Brasso WB, Anderson K, Lonsway DR, Pierce VM, et al. Multicenter evaluation of the modified carbapenem inactivation method and the Carba NP for detection of carbapenemase-producing Pseudomonas aeruginosa and Acinetobacter baumannii. J Clin Microbiol 2017; 56: e01369-17. https://doi.org/10.1128/JCM.0136917.

23. Tamma PD, Goodman KE, Harris AD, Tekle T, Roberts A, Taiwo A, et al. Comparing the outcomes of patients with carbapenemase-producing and non-carbapenemaseproducing carbapenem-resistant Enterobacteriaceae bacteremia. Clin Infect Dis 2017; 64: 257-264. https://doi.org/ 10.1093/cid/ciw741. 